\title{
KEY ASPECTS OF DENTAL DIAGNOSTICS AND TREATMENT SPECIFICS IN ECTODERMAL DYSPLASIA PATIENTS: COMPREHENSIVE LITERATURE REVIEW
}

\author{
Myroslav Goncharuk-Khomyn' ${ }^{1}$, Izzet Yavuz ${ }^{2}$, Alessandro L. Cavalcanti ${ }^{3}$, Alina Boykiv ${ }^{4}$, Yaroslav Nahirnyi ${ }^{5}$ \\ 'Department of Prosthetic Dentistry, Uzhhorod National University, Ukraine \\ 2Department of Pedodontics, Dicle Universiry, Turkey \\ ${ }^{3}$ Department of Dentistry, State University of Campina Grande-Paraiba, Brazil \\ ${ }^{4}$ Department of Orthopedic Dentistry, I. Horbachevsky Ternopil National Medical University, Ukraine \\ ${ }^{5}$ Department of Surgical Dentistry, I. Horbachevsky Ternopil National Medical University, Ukraine
}

\begin{abstract}
InTroduction: Pathogenesis of ectodermal dysplasia (ED) is explained by alterations occurring within derivates of ectoderm, caused by different genetic violations.

ОвјестіVEs: The aim of this study was to provide critical comprehensive literature review considering diagnostic and treatment specifics among dental patients with ED.

MATERIAL AND METHODs: Comprehensive literature search was provided via PubMed database (https://pubmed. ncbi.nlm.nih.gov/) and Google Scholar search engine (https://scholar.google.com/). Systematized number of publications was evaluated as a subject of manual content analysis, categories of which included dental diagnostic and treatment aspects of patients with ED.

RESULTS: Treatment approaches for remaining deciduous and permanent teeth under the condition of future prosthodontic rehabilitation planning differ in numbers of publications dedicated to the topic of dental care provision among ED patients. Such non-consistency could be explained by diversities in described prosthetic rehabilitation protocols, and initial variations of teeth's form, structure, developmental stages, and their overall quantity registered during primary examination of ED dental patients, including possible alterations in enamel integrity, dispersions in pulp chamber volume, caries intensity levels, changes in root formation stages, and eruption phases during ED.

ConcLusions: The most prominent dental and oral signs of ED that potentially could be used as criteria for diagnostics with varying degree of diagnostic significance, include oligodontia, delayed eruption and development of teeth, changed tooth crown and/or root morphology, decreased salivary function, cleft lip and/or palate. However, there is no unified algorithm of ED dental patients' rehabilitation, and planning of such should consider a number of initial clinical situation's factors, ED manifestations, individual functional prognosis, and esthetic demands.
\end{abstract}

KEY wORDS: ectodermal dysplasia, dental treatment, diagnostics, oral manifestations, review.

J Stoma 2020; 73, 6: 342-350

DOI: https://doi.org/10.5114/jos.2020.102053

\section{JOURNAL OF STOMATOLOGY CZASOPISMO STOMATOLOGICZNE

ADDRESS FOR CORRESPONDENCE: Myroslav Goncharuk-Khomyn, Department of Prosthetic Dentistry, Uzhhorod National University, Univesitetska st., 88000, Uzhhorod, Ukraine, e-mail: myroslav.goncharuk-khomyn@uzhnu.edu.ua

ReCEIVED: 29.07.2020 • ACCEPTED: 07.11.2020 • Published: 30.12.2020 


\section{INTRODUCTION}

Pathogenesis of ectodermal dysplasia (ED) is explained by alterations occurring within derivates of ectoderm, caused by different genetic violations [1-3]. Due to data presented in 1995, the prevalence of ED has been estimated at a range of 1 on 10,000-100,000 live births (considering just males) [1]. Nguyen-Nielsen et al. demonstrated that the prevalence of ED is influenced by the method used for its diagnostics [2]. Usage of just molecularly-oriented laboratorial approaches helped to register 1.6 pathology cases per 100,000 subjects, while additional use of clinical symptoms increased this number to 4.2 cases [2]. Generally, in 2013, results of a population-based study demonstrated overall cumulative prevalence of 21.9 ED cases among 100,000 people [2]. Main risks of ED spread among family members is related to de novo carrier or link-to-link mechanisms [3].

Generally, three main types of ED have been identified, which include hidrotic (also called "Clouston syndrome"), anhidrotic (also called "Christ-SiemensTouraine syndrome"), and hypohidrotic $[3,4]$. Due to the Freire-Maia's classification, group A includes minimum two structural defects among ectodermal derivates, such as hair, nails, teeth, and sweat glands, while group B represents only one ectodermal derivate defect among the above-mentioned, with one additional defect [5]. In a populational-based study, it was found that dental abnormalities were the most prevalent and correlated major symptoms, with gradually increasing frequency among molecularly identified, clinically diagnosed, and suppositional ED patients, with $79.4 \%$ prevalence over all study sample [2]. Moreover, dental dysplasia has been described as one of the four main defects associated with ED pathology [3].

As per international conference statement, the perspectives of ED dental treatment studies are related to specific rehabilitation algorithm, which analyses already existing approaches and their effectiveness in the means of esthetic parameters, functional criteria, and quality of life [6].

\section{MATERIAL AND METHODS}

Comprehensive literature search was performed via PubMed database (https://pubmed.ncbi.nlm.nih. gov/) and Google Scholar search engine (https://scholar.google.com/). Search algorithm using MeSH terms in PubMed was done as follow: ("ectodermal dysplasia”[MeSH Terms] OR ("ectodermal”[All Fields] AND "dysplasia”[All Fields]) OR “ectodermal dysplasia”[All Fields]) AND ("dental health services"[MeSH Terms] OR ("dental"[All Fields] AND "health"[All Fields] AND "services"[All Fields]) OR "dental health services"[All Fields] OR "dental”[All Fields]) [7]. For Google Scholar search, "ectodermal dysplasia", "dental", "dental treat- ment", "teeth", “oral cavity" as keywords were used [8]. Criteria of publication date was not used. Publication in English or at least with English annotations were included in the final sample. Systematized number of publications was evaluated as a subject of manual content analysis, categories of which included dental diagnostic and treatment aspects of patients with ED. Microsoft Excel 2019 (Microsoft Office, 2019) software was applied for obtained results classification to appropriate interpret the obtained findings.

\section{RESULTS AND DISCUSSION}

\section{RESULTS OF LITERATURE SEARCH}

In total, 1,169 articles were found, including 804 papers from PubMed and 365 from Google Scholar. After removing duplicates, 889 publications remained, of which 156 were selected by reading titles and abstracts. Other 733 articles were excluded, because they were not associated with the topic of ED impact in dentistry. All of 156 articles identified were completely analyzed. After full reading, 74 articles were excluded because of not providing information on the purpose of review. Out of 82 completely eligible articles, 34 were excluded due to repeating results or demonstrating analogic data presented in other articles. At the end, 48 articles were included in this review, with 15 case reports and case series (31.25\%), 13 reviews, including 6 systematic reviews (27.09\%), 1 cross-sectional study $(2.08 \%)$, 16 retrospective $(33.34 \%)$ and 1 prospective $(2.08 \%)$ studies, 1 paper with practical guidelines in the form of international conference statements (2.08\%), and 1 article from the website of National Foundation for Ectodermal Dysplasia (2.08\%) (Figure 1).

\section{ORAL AND DENTAL SIGNS OF ECTODERMAL DYSPLASIA}

Among different oral and dental signs of ED, several indicators were mentioned as the most prevalent and clinically prominent (Figure 2).

In Hanisch et al. publication, it was mentioned that mono-symptomatic cases of ED are also possible and already reported in literature [9].

Dogan reported dental anomalies in $100 \%$ of studied ED cases [5]. Among other 23 already diagnosed ED patients, a fundamental indicator in the form of tooth agenesis was registered also within $100 \%$ of subjects, while other non-dental signs revealed $43.5-91.3 \%$ prevalence [10]. In a retrospective study of 15 cases with ED, authors stated that $100 \%$ of investigated subjects suffered from hypodontia [11]. Açikgöz et al. described an ED patient with true full deciduous teeth adentia, in whom radiography revealed the presence of only 

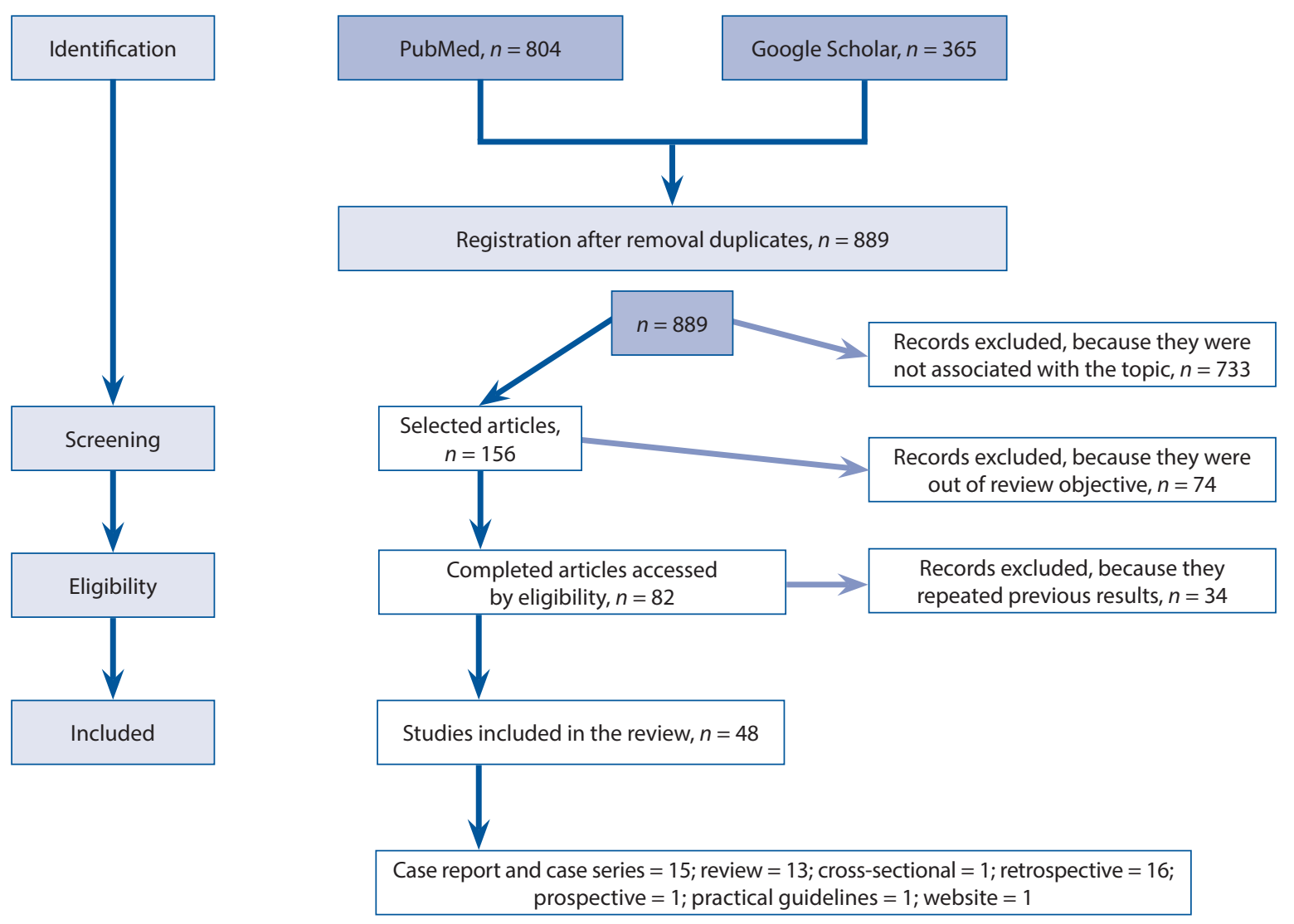

FIGURE 1. Flowchart of selection procedure for the articles included in the review

Oligodontia ("as stronger clinical expression of hypodontia"[4])

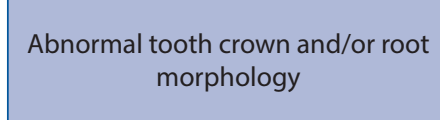

Enamel hypoplasia
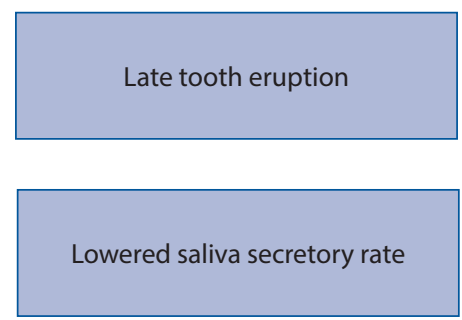

Cleftlip and/or palate
Tooth agenesis

Malformations of salivary gland (with unknown prevalence)

Oral leukokeratosis

FIGURE 2. Most prevalent and clinically prominent oral and dental signs of ectodermal dysplasia [4]

unerupted permanent canines [12]. Later, analogical case was described with full adentia in hypohidrotic ED [13]. Another clinical case was published with full primary and secondary anodontia of an 8-year-old patient with ED [14]. It is interesting that in previous studies, authors also had mentioned that essential adentia within primary dentition during ED occurs comparatively less often than within permanent dentition $[4,11,12]$. Among permanent dentition lateral incisors, second premolars, first premolars, and canines were described as having the greatest chance of being missed during ED pathology $[4,11]$. An interested fact was noted during study on 16 children with ED, i.e., bilateral teeth agenesis pattern, which was observed on maxilla with nearly $94 \%$ frequency and on mandible with $75 \%$ frequency, while greater percentage of missed teeth was observed on mandible [15]. Mean number of missed permanent teeth among males (22) was greater compared to females (4) [4]. Considering such data, it was emphasized that oligodontia itself could be used as an identification marker, which could suggest the presence of ED [16].

A recent retrospective study of $49 \mathrm{ED}$ patients revealed that oligodontia and mandibular protrusion was associated with $100 \%$ of study subjects, while tooth crown and 
root anomalies were observed in $75.51 \%$ and $6.12 \%$ of cases, respectively [17]. Analyses of 36 patients provided by Dogan showed that only $69.4 \%$ of patients were characterized with altered teeth form (conical teeth), whereas abnormal root formation was noted in $8.3 \%$ of cases, external root resorption in $2.7 \%$, and abnormal root shape in $8.3 \%$ of cases [5]. In another research, altered tooth crown morphology was noted among $100 \%$ of analyzed male subjects and in $84 \%$ of female patients, and males were also characterized with either maxillary lateral incisors, maxillary first premolars, mandibular incisors, or mandibular premolars being initially absent in $100 \%$ of analyzed ED cases [4]. Changes in a tooth form and structure, which could be clinically noted and associated with ED alterations, increased the risk of fatal primary dentition root resorption by 1.46 compared to healthy patients' sample [18]. Also, female pediatric patients were characterized with higher frequency of possible root malformations (pyramid-shaped form, fusion, dilaceration) compared to male sample with ED pathology, while gender relationship due to the tooth crown abnormalities was reversed [15].

In three case reports, a risk of enamel caries development and microbiological changes within oral cavity during ED was associated with alterations of normal salivary parameters [19].

Study by Turkish researchers showed not only the presence of tooth quantity, shape, and structure differences between healthy sample and ED patients, but disparities within orthodontic parameters were noted considering results of Steiner, McNamara, Subtelyn, and Ricketts analyses [5]. Steiner's results on lateral cephalometries were considered to be a useful tool for orthodontic evaluation in ED patients; however, the severity of ED manifestations should be attentionally observed during dental diagnostics $[11,17]$. Dellavia et al. noted a reduced pattern of facial growth among patients with ED with nearly 2 years delay compared to the normal jaws peek development period [20]. Among 15 subjects with ED, 53.3\% presented with different orthodontic deviations, which included reduction of facial height, hypotrophy and retrusion of maxilla, mandibular protrusion with upward displacement, and skeletal class III malocclusion [11]. Moreover, the authors emphasized a relationship between orthodontic-associated deviations with the severity of hypodontia manifestations [11].

In a retrospective study, it was shown that among 19 analyzed ED cases, $100 \%$ of patients presented with insufficient thickness of alveolar ridge [21]. Bone mineral density evaluation of mandible among ED patients using a Hounsfield unit, revealed a statistically lower value compared to healthy control group [22]. These findings could be used not only as criteria for ED diagnostics, but could be applied during treatment planning of ED patients in prosthetics design with respective support for dental implants. Nevertheless, in other study, no difference of trabecular mandibular structure was noted between healthy and ED subjects [23]. Use of CBCT helped to understand that maxillary sinus volume and its surface area among patients with ED were statistically smaller compared to control group of individuals without the above-mentioned pathology [24].

Loss of vertical occlusal dimension during ED was associated with altered development of maxilla bones (anatomical retrusion) and dislocation of mandible bone. This should be greatly appointed during prosthetic treatment planning of ED patients, with the aim to prevent possible oral functional complications of iatrogenic origin. Articulation problems were also described as one of the possible dental features during ED.

In Rosa et al. study, the authors have not found any statistically significant difference between registered pattern of dental abnormalities and analyzed type of ED [25]. Analogically correlation between hypodontia, patient's sex, and cleft's type were not statistically evidenced [25]. However, in some studies, specific dental phenotypes related to the genotype of ED were described [19]; treatment planning among patients with different phenotypes still should be based on individual disease features and diagnosed dental manifestations.

Female dental patients have been comparatively less often affected by ED pathology than male patients [3]. Moreover, in Hanisch et al. study, male patients were reported of being diagnosed with ED earlier compared to females [9]. Also, in Danish populational study, peak diagnostic age of ED among males was 11.1 years, while for females, it was 11.3 years, based on an analysis of molecularly identified and clinically diagnosed cases [2].

\section{DENTAL TREATMENT SPECIFICS OF PATIENTS WITH ECTODERMAL DYSPLASIA: PROSTHETIC APPROACHES}

Due to specificities of dental ED patients' rehabilitation, a list of relevant dental dataset was proposed in order to support dental treatment optimization and planning organization [6].

Literature review provided by Pigno et al. in 1996 noted three possible prosthetic rehabilitation perspectives for patients with $\mathrm{ED}$, such as fixed prosthodontics, removable dentures, and implant prosthodontics [26]. In this review, the author mentioned that the use of fixed prosthodontics could not greatly interfere with the growth of jawbone (it should be avoided in young patients with active growth stage), but dentists should pay attention to pulp size parameters during teeth preparations [26]. Later, prosthetic considerations were added to overdentures as specific type of rehabilitation, which were described in the following situations: 1 . Increased retention; 2 . Residual ridge and tooth structures preservation; 3 . Improved restoration of masticatory function and ability [27]. Due to the available information, primary prosthetic treatment could be initiated as early as for 2-, 3-, or 4-year-old patients. The National Foundation for Ectodermal Dysplasia' guidelines also considered complete dentures or 
overdentures for pediatric patients of 0-6 years old [28]. Early prosthetic treatment in ED dental patients could help to prevent further atrophy of bone ridges.

Bal et al. mentioned that dental care needed for pediatric ED patients should be supplied with their growing pattern, considering age-related changes of functional tendencies, and esthetic demands [29]. As an option of temporary and reversible rehabilitation method, the researchers proposed the use of removable partial dentures with direct photo-composite restorations [29]. In a number of cases, removable dentures in ED patients were used in prosthetic rehabilitation or they were primary considered as temporary treatment modality, which could be achieved in early childhood, as definitive implant-associated approach could be postponed [30, 31].

Combination of partial and complete dentures can be used for ED patients with different intensity of oligodontia/adentia on maxilla and mandible. Removable partial dentures itself can be used as a treatment option, while in some cases, they support the restoration of vertical height parameter followed by definitive overlay removable partial denture. Using conventional dentures complies with the normal developmental growth pattern of both jaws, which also stimulates adequate morphology retention of their bone ridges. This approach supports further dental implant treatment planning.

Maroulakos et al. proposed an algorithm, which aimed at an optimization in choosing between removable partial denture or overdenture as a treatment option for ED patients [32]. The absence of posterior teeth, deficiency of facial esthetic parameters, need for the lip support, lack of sufficient quantity of teeth, and loss of vertical occlusal dimension were the factors contributed to the choice of overdenture as a treatment option [32]. Additionally, soft liner and tissue-supported overdentures can be used as prosthetic rehabilitation designs for ED patients with severe form of oligodontia.

Systematic review of prosthetic treatments in dental ED patients emphasized several important rehabilitation aspects for different age periods (Figure 3) [33].

\section{ROLE AND PROGNOSIS OF DENTAL IMPLANTS USED FOR THE REHABILITATION OF ECTODERMAL DYSPLASIA PATIENTS}

The results of a systematic review on different prosthetic treatment methods in ED patients showed that the mean survival rate of implants was $95.3 \%$, tooth

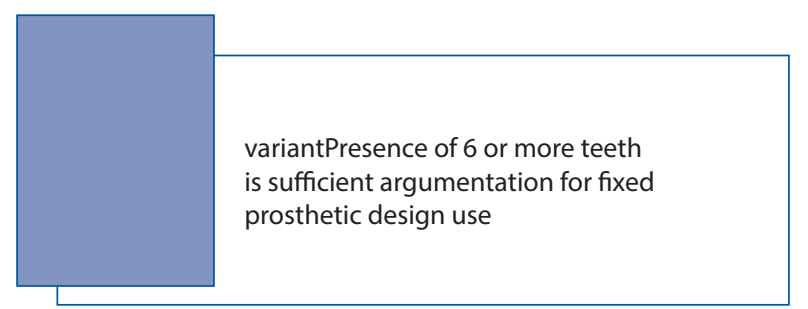
considered prosthetic construction for oligodontia patients, while overdentures represent alternative variant
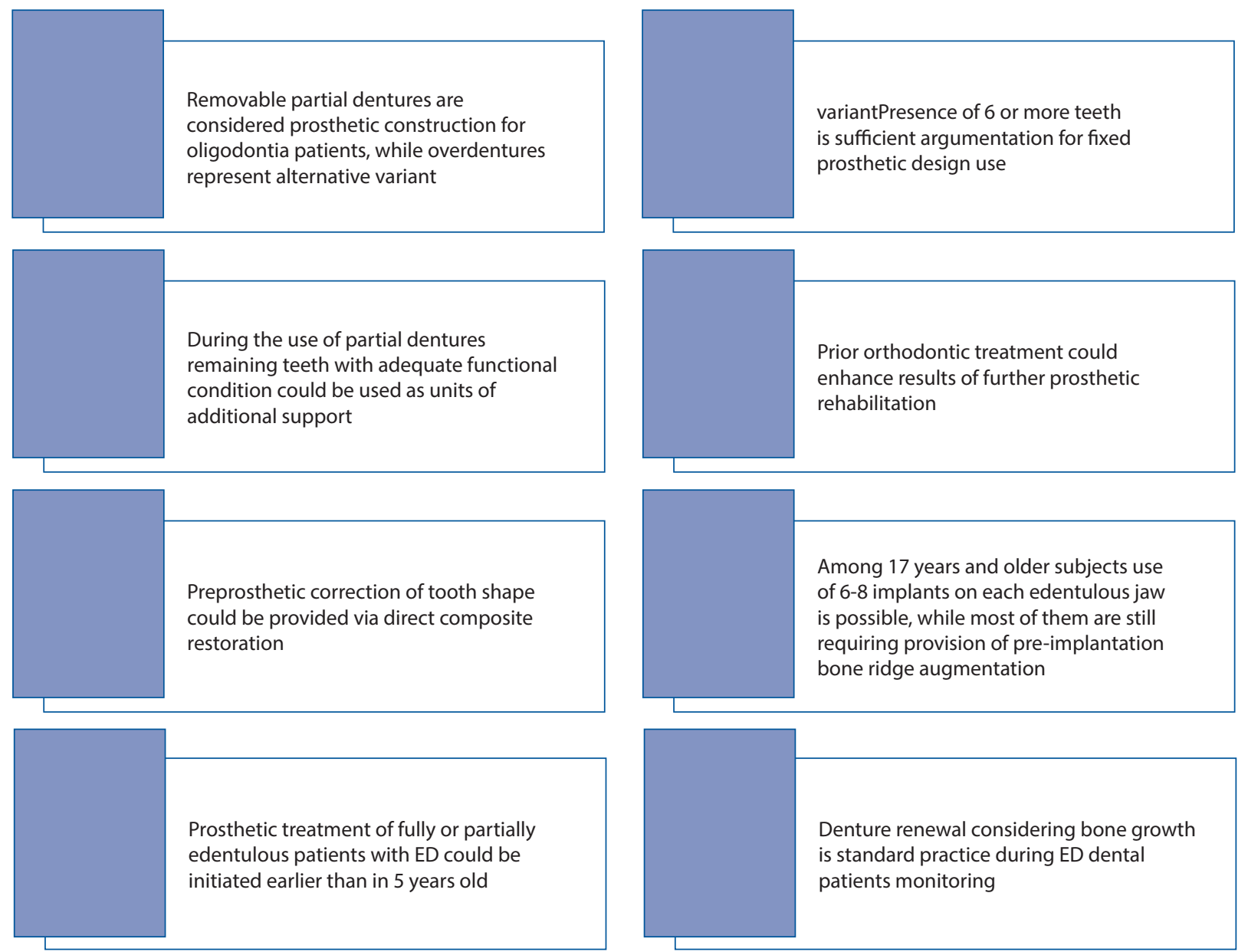

FIGURE 3. Prosthetic rehabilitation aspects to be considered in patients with ectodermal dysplasia [33] 
TABLE 1. Data on dental implants survival rates among ectodermal dysplasia dental patients

\begin{tabular}{|l|c|c|}
\hline Publication (year) & Type of study & Survival/ success/ failure rates \\
\hline Terheyden and Wüsthoff (2015) & Systematic review & Survival rate: $95.3 \%$ \\
\hline Bergendal et al. (2008) & Retrospective study & Failure rate: $35.7-93.9 \%$ \\
\hline Filius et al. (2016) & Systematic review & Survival rate: $35.7-98.7 \%$ (mean, $93.7 \%)$ \\
\hline Wang et al. (2016) & Systematic review & Survival rate: $97.9 \%$ (among adults), $98.6 \%$ (among children) \\
\hline Chranovic (2018) & Systematic review & Survival rate: $84.6 \%$ \\
\hline
\end{tabular}

autotransplants accounted for $94.4 \%$, and conventional prosthetic constructions was observed as $60.2 \%$ [34]. The highest level of satisfaction with a treatment was also reported after the use of dental implants (93.4\%), while for conventional prosthodontic constructions, tooth autotransplants, and orthodontic space closure appliances, these parameters were lower but relatively analogical (76.6\%, 72.0\%, and 65.5\%, respectively) [34].

Retrospective analyses among Swedish children, who were rehabilitated with the use of dental implants, has shown that failure rate of intraosseous fixtures among pediatric patients with ED could decrease to $64.3 \%$ [35]. However, authors mentioned that not ED itself, but primary clinical condition as well as compromised jaw size were the main risk factors associated with implant loss [35]. Later, the same author highlighted that ED patients could have compromised dental implants survival rates due to mutations influence on bone structure [16].

A systematic review of prosthetic treatment results among patients with severe hypodontia, which included patients with ED, shown that the range of implant survival was 35.7-98.7\% (mean, 93.7\%) [36]. Higher rate of implant loss among ED patients compared to healthy subjects was due to deficiency of bone quality and quantity, but not with the ED pathology itself, which was not a contraindication for dental implantation [36].

Another systematic review of Wang et al. had shown that implant survival rates reached $97.9 \%$ and $98.6 \%$ among adults and children with ED, respectively, while success rate among adults was registered at a level of $93.7 \%$, with nearly 24 months of follow-up [37].

Yet another systematic review provided by Chranovic reported that cumulative survival rate of dental implant placed among ED patients reached $84.6 \%$ over 20 years of follow-up, with a mean of nearly 8 implants received by each patient [38] (Table 1).

Considering compromised conditions of bone proposal among dental ED patients, Mello et al. described an effective approach of prosthetic rehabilitation with the use of mini implants [39].

Kirmeier et al. suggested that dental implants' placement accompanied by bone augmentation and bone deficiency due to hypohidrotic ED, could be categorized as an adaptive variant of rehabilitation, if XLHED gene alteration was identified at the first place [40]. However, it should be mentioned that the authors used a delayed protocol of implantation, with further bar-retained overdentures for prosthetic rehabilitation [40]. Possibility of implant immediate loading in adult patients with ED was also reported [41].

\section{SPECIFICS OF IMPLANT-PROSTHETIC REHABILITATION AMONG ECTODERMAL DYSPLASIA DENTAL PATIENTS}

In both systematic reviews described above, the authors mentioned that the choice of ideal time for implant installation with rehabilitation purpose among children with ED, remains debatable $[37,38]$. Due to this, Wang et al. highlighted the importance of skeletal maturity evaluation before implant placement as one of the indicators of possible requirement for this procedure, and patient-specific factors as another one [37]. The National Foundation for Ectodermal Dysplasia recommended dental implantation as a variant of treatment in pediatric patients older than 7 years, for only anterior mandible area [28]. Such parameters as stage of skeletal growth, conditions of present dental status, number of missed teeth, level of technical support, and psychological patients' status should be considered as relevant parameters before ED patients' rehabilitation with the use of dental implants $[37,38]$.

No significant influence of implant installation on jawbone growth was noted in previous study, pattern of which was relatively analogical between ED children, who received dental implant and those who had not. Triches et al. emphasized the specifics of maxilla and mandible growth, which should be considered during implant placement in dental ED patients [42]. It should be noted that enlargement of maxilla during growth progress occurs through surface remodeling and bone appositions in downward and forward directions, while mandible is characterized with backward and upward condyle growth and backward growth of the ramus. Taking that into account, the placement of implant in frontal maxillary region should be avoided, since this area presents with prevalent resorption pattern, whereas mandibular frontal area reaches stable position much earlier [42]. Growth analysis of jaws among ED patients with installed dental implants demonstrated that maxillary intraosseous fixtures were characterized with minor impaction, while mandibular ones showed a different 
TABLE 2. Time-structured treatment algorithm for ectrodactyly ectodermal dysplasia-cleft syndrome patients proposed by Rachimel et al. [46]

\begin{tabular}{|l|l|l|}
\hline Stage & Procedure & Age period \\
\hline I & Restoration of lip and palate defects & During infancy and early childhood periods \\
\hline II & Secondary reconstruction of alveolar defect by bone grafting & For 8-11 years old period \\
\hline III & Expansion of maxilla with the use of distraction osteogenesis method & Following puberty period \\
\hline IV & Maxillary and ridge bone augmentation based on possible need & Adulthood period \\
\hline V & Dental implantation and final prosthetic treatment & Adulthood period \\
\hline
\end{tabular}

TABLE 3. Oral care supply program for the patients with hypohidrotic ectodermal dysplasia [47]

\begin{tabular}{|c|c|}
\hline Age phase & Targeted approach \\
\hline Childhood phase & To retain the function and effectiveness of firstly manufactured prostheses \\
\hline Adolescent phase & To prepare patient for future permanent and final rehabilitation \\
\hline Adult phase & All the dental interventions should be oriented on definitive prosthetic treatment \\
\hline
\end{tabular}

inclination [43]. Such outcomes were related to the nasal and sinus floor relocation within growing maxilla and rotation effect during mandible growth [43].

Even though results of a survey showed that nearly $50 \%$ of ED patients with dental implants demonstrated different kind of complications, and 24\% were observed with some type of unsuccessful implant treatment outcomes, more than $90 \%$ of the total number of respondents were satisfied with their implant rehabilitation in terms of time, cost, and effectiveness [44].

\section{ADDITIONAL, SUPPORTIVE, AND PHASED APPROACHES OF DENTAL TREATMENT USED FOR ECTODERMAL DYSPLASIA PATIENTS}

Orthodontic support and efficient treatment can form conditions for optimal maxillofacial growth while adapting favorable inter-jaw association [45]. Surgical approaches, which possibly can differ among patients with ED include orthognathic management, midfacial defects correction, cleft lip or/ and palate repair, and supportive augmentation procedure, with optimizing condition for future dental implants placement [46].

Rachimel et al. proposed time-structured treatment algorithm for ectrodactyly ED-cleft syndrome patients with severe atrophic mandible, which included next stages [46] (Table 2).

Specially developed oral care supply program was presented for the patients with hypohidrotic ED, categorized due to the different age periods and dentition status [47]. Implementation of such program could effectively optimize the approach for predictive dental treatment, considering age-related changes of maxillofacial system (Table 3).

Continuing growth of maxillofacial structure among dental ED patients reveals the need for constant moni- toring with the aim of possible treatment correction that could optimize the final rehabilitation results.

Nevertheless, multidisciplinary approach remains one of the most predictive variants of ED dental patients' management. In analyses of ED case series authors pointed on the need of protective dental treatment considering remaining teeth in the oral cavity with the aim of preventing their possible loss in future [48]. Moreover, due to the international conference statements multidisciplinary approach in the first please includes sufficiently organized prophylaxis strategies, while also considering the need of ineffective treatment exclusion [6].

Hanisch et al. reported that OHIP (oral health-related quality of life) values among patients with ED were ones of the highest (mean, $15.32 \%$ ), while satisfaction of such patients with dentist was also one of the greatest (97.61\%) [49]. In contrast, satisfaction of such patients with health system was one of the lowest and reached just 15.56\%, which was interpreted by the author as possibility for improvement by further incorporation of dental implants as possible rehabilitation offer [49]. Due to the retrospective study of among ED patients, lower quality of life parameter was noted among patients who had trouble of finding corresponding dentist, even though $72.65 \%$ of subjects responded as those who already had found appropriate dental specialist [9]. Even after effective dental treatment of ED patients' enhancement of quality of life parameters could be reached with further reconstructive iatrogenic interventions, considering possible hypertrophic lip or lipofilling correction and rhinoplasty [50].

\section{LIMITATIONS AND CLINICAL IMPACT OF REVIEW}

Limitations of the present study is due to it retrospective literature review design, which was unable to pro- 
vide bias analysis and quantitative statistical comparison of several targeted numbered parameters, but the primary objective of this study was originally dedicated to gain relevant data on diagnostic and treatment aspects of ED patients during their dental rehabilitation. Moreover, dental treatment approaches are still developing, focusing on mini-invasive parameters, even with multidisciplinary rehabilitation, so differentiation of treatment concepts during previous few years remains the area of interest. Therefore, a comprehensive literature review could be considered as the first step in data collection for future systematic review with corresponding analyses, as the most relevant and objective report could provide the most up-to-date fact-finding research.

It was found that approaches of remaining deciduous and permanent teeth treatment under the conditions of future prosthodontic rehabilitation planning differs in number of publications dedicated to the topic of dental care provided for ED patients. Such non-consistency could be explained by the diversities in described prosthetic rehabilitation protocols, and initial variations of teeth forms, structures, developmental stages, and their overall quantity registered during primary examination of dental ED patients, including possible alterations in enamel integrity, dispersions in pulp chamber volume, caries intensity levels, changes in root formation stages, and eruption phases during ED. Considering these findings, future research could focus on objectification of each tooth condition, since such approaches were previously described in the literature with different treatment algorithm selection. Moreover, it was revealed that severity of oligodontia related to the degree of craniofacial growth alterations. Based on this fact, early objective assessment of dental status and each tooth condition potentially could be used as a predictive parameter for further evaluation of needed maxillofacial growth correction. Furthermore, the future interest might be dedicated to the evaluation of dental age parameters among ED patients and their relation to skeletal maturation phase since inter-relation of such aspects greatly influence periodization and predictiveness of dental treatment.

\section{CONCLUSIONS}

Most of the studies were dedicated to the aspects of dental ED patients' diagnostics and treatment, and were presented in the form of case reports, case series reports, and retrospective and prospective observational studies, while the deficiency of systematic review and meta-analyses was noted. Nonetheless, few studies with the highest evidence-based level have been recognized, which mostly deemed prosthetic and implant treatment outcomes of dental ED patients. Conditions for providing meta-analysis considering effectiveness of different dental treatment protocols are limited, since analyzed publication characterized with variations of target re- search parameters and long-term results in most of published articles are absent. The most prominent dental and oral signs of ED that potentially could be used as criteria for diagnostics with varying degree of diagnostic significance include oligodontia, delayed eruption and development of teeth, changed tooth crown and/or root morphology, decreased salivary function, cleft lip, and/ or palate. Still, there is no unified algorithm of dental ED patients' rehabilitation, and planning of such should consider a number of initial clinical factors, ED manifestations, individual functional prognosis, and esthetic demands.

\section{CONFLICT OF INTEREST}

The authors declare no potential conflicts of interest with respect to the research, authorship, and/or publication of this article.

\section{References}

1. Kupietzky A, Houpt M. Hypohidrotic ectodermal dysplasia: characteristics and treatment. Quintessence Int 1995; 26: 285-291.

2. Nguyen-Nielsen M, Skovbo S, Svaneby D, Pedersen L, Fryzek J. The prevalence of X-linked hypohidrotic ectodermal dysplasia (XLHED) in Denmark, 1995-2010. Eur J Med Genet 2013; 56: 236-242.

3. Deshmukh S, Prashanth S. Ectodermal dysplasia: a genetic review. Int J Clin Pediatr Dent 2012; 5: 197-202.

4. Bergendal B. Orodental manifestations in ectodermal dysplasia a review. Am J Med Genet A 2014; 164: 2465-2471.

5. Doğan MS, Callea M, Yavuz İ, et al. An evaluation of clinical, radiological and three-dimensional dental tomography findings in ectodermal dysplasia cases. Med Oral Patol Oral Cir Bucal 2015; 20: e340-e346.

6. Hobkirk JA, Nohl F, Bergendal B, Storhaug K, Richter MK. The management of ectodermal dysplasia and severe hypodontia. International conference statements. J Oral Rehabil 2006; 33: 634-637.

7. Fatehi F, Gray LC, Wootton R. How to improve your PubMed/ MEDLINE searches: 3. advanced searching, MeSH and My NCBI. J Telemed Telecare 2014; 20: 102-112.

8. Walters WH. Google Scholar search performance: comparative recall and precision. portal: Libraries and the Academy 2009; 9: 5-24.

9. Hanisch M, Sielker S, Jung S, Kleinheinz J, Bohner L. Self-assessment of oral health-related quality of life in people with ectodermal dysplasia in Germany. Int J Environ Res Public Health 2019; 16: 1933.

10. Yildirim M, Yorgancilar E, Gun R, Topcu I. Ectodermal dysplasia: otolaryngologic evaluation of 23 cases. Ear Nose Throat J 2012; 91: E28-33.

11. Yavuz I, Baskan Z, Ulku R, et al. Ectodermal dysplasia: retrospective study of fifteen cases. Arch Med Res 2006; 37: 403-409.

12. Açıkgöz A, Kademoglu O, Elekdag-Türk S, Karagöz F. Hypohidrotic ectodermal dysplasia with true anodontia of the primary dentition. Quintessence Int 2007; 38: 853-858.

13. Sholapurkar AA, Setty S, Pai KM. Total anodontia in patient with hypohidrotic ectodermal dysplasia. Report of rare case of Christ-Siemens Touraine syndrome. N Y State Dent J 2011; 77: 36-39.

14. Bala M, Pathak A. Ectodermal dysplasia with true anodontia. J Oral Maxillofac Pathol 2011; 15: 244-246.

15. Tuna EB, Guven Y, Bozdogan E, Aktoren O. Assessment of dental features in 16 children with hypohidrotic ectodermal dysplasia. Pediatric Dental Journal 2009; 19: 106-111. 
16. Bergendal B. Oligodontia ectodermal dysplasia - on signs, symptoms, genetics, and outcomes of dental treatment. Swedish dental journal. Supplement 2010, 13-78.

17. Dogan MS, Atas O, Yavuz İ, Tekin S. Ektodermal Displazili Olgularda Klinik ve Radyolojik Bulguların İncelenmesi. Firat Tip Dergisi 2019; 24: 85-88.

18. Kjær I, Nielsen MH, Skovgaard LT. Can persistence of primary molars be predicted in subjects with multiple tooth agenesis? Eur J Orthod 2008; 30: 249-253.

19. Gomes MF, Sichi LG, Giannasi LC, et al. Phenotypic features and salivary parameters in patients with ectodermal dysplasia: report of three cases. Case Reports in Dentistry 2018; 2018: 2409212.

20. Dellavia C, Catti F, Sforza C, Tommasi DG, Ferrario VF. Craniofacial growth in ectodermal dysplasia: an 8 year longitudinal evaluation of Italian subjects. Angle Orthod 2010; 80: 733-739.

21. More CB, Bhavsar K, Joshi J, Varma SN, Tailor M. Hereditary ectodermal dysplasia: a retrospective study. J Nat Sci Biol Med 2013; 4: 445-450.

22. Çolak M. An evaluation of bone mineral density using cone beam computed tomography in patients with ectodermal dysplasia: a retrospective study at a single center in Turkey. Med Sci Monit 2019; 25: 3503-3509.

23. Créton M, Geraets W, Verhoeven JW, van der Stelt PF, Verhey H, Cune M. Radiographic features of mandibular trabecular bone structure in hypodontia. Clinical Implant Dentistry and Related Research 2012; 14: 241-249.

24. Yalcin ED, Koparal M, Aksoy O. The effect of ectodermal dysplasia on volume and surface area of maxillary sinus. Eur Arch Otorhinolaryngol 2018; 275: 2991-2996.

25. Rosa RR, Janeiro MM, Camargo SE, Porto CD, Kreich EM, Henriques JC. Radiographic study of patients with ectodermal dysplasia and partial. Indian Journal of Dental Research 2012; 23 801-805.

26. Pigno MA, Blackman RB, Cronin RJ, Cavazos E. Prosthodontic management of ectodermal dysplasia: a review of the literature. Journal of Prosthetic Dentistry 1996; 76: 541-545.

27. Kumari N, Kumar G, Christopher Sunil D, Anto N. Ectodermal dysplasia: prosthodontic considerations. Int J Oral Health Med Res 2016; 3: 92-94.

28. National Foundation for Ectodermal Dysplasias. Available at: https://www.nfed.org/ (Accessed: 21.06.2020).

29. Bal C, Bal BT, Tüfekçioğlu D. Treatment considerations for a patient with hypohidrotic ectodermal dysplasia: a case report. Journal of Contemporary Dental Practice 2008; 9: 128-134.

30. Bidra AS, Martin JW, Feldman E. Complete denture prosthodon tics in children with ectodermal dysplasia: review of principles and techniques. Compend Contin Educ Dent 2010; 31: 426-433.

31. Bidaki M, Moghadam NC, Takhtdar M, Kamareh S, Kordafshari B. Complete denture prosthodontics in children with ectodermal dysplasia: systematic review from case reports and case series reports. Journal of International Pharmaceutical Research 2019; 46: 181-184.

32. Maroulakos G, Artopoulou II, Angelopoulou MV, Emmanouil D. Removable partial dentures vs overdentures in children with ectodermal dysplasia: two case reports. Eur Arch Paediatr Dent 2016; 17: 205-210

33. Schnabl D, Grunert I, Schmuth M, Kapferer-Seebacher I. Prosthetic rehabilitation of patients with hypohidrotic ectodermal dysplasia: a systematic review. J Oral Rehab 2018; 45: 555-570.

34. Terheyden H, Wüsthoff F. Occlusal rehabilitation in patients with congenitally missing teeth - dental implants, conventional prosthetics, tooth autotransplants, and preservation of deciduous teeth - a systematic review. Int J Implant Dent 2015; 1: 30.

35. Bergendal B, Ekman A, Nilsson P. Implant failure in young children with ectodermal dysplasia: a retrospective evaluation of use and outcome of dental implant treatment in children in Sweden. Int J Oral Maxillofac Implants 2008; 23: 520-524

36. Filius MA, Cune MS, Raghoebar GM, Vissink A, Visser A. Prosthetic treatment outcome in patients with severe hypodontia: a systematic review. J Oral Rehab 2016; 43: 373-387.
37. Wang Y, He J, Decker AM, Hu JC, Zou D. Clinical outcomes of implant therapy in ectodermal dysplasia patients: a systematic review. Int J Oral Maxillofac Surg 2016; 45: 1035-1043.

38. Chrcanovic BR. Dental implants in patients with ectodermal dysplasia: a systematic review. J Craniomaxillofac Surg 2018; 46 : 1211-1217.

39. Mello BZ, Silva TC, Rios D, Machado MA, Valarelli FP, Oliveira TM. Mini-implants: alternative for oral rehabilitation of a child with ectodermal dysplasia. Braz Dent J 2015; 26: 75-78.

40. Kirmeier R, Gluhak C, Marada P, Wegscheider WA, Eskici A, Jakse $\mathrm{N}$. Oral rehabilitation of adult twins with severe lack of bone due to hypohidrotic ectodermal dysplasia - a 12-month follow-up. J Oral Maxillofac Surg 2009; 67: 189-194.

41. D’Ambrosio A, Cioffi A, Cirillo A, Sammartino G. Immediate loading in ectodermal dysplasia: clinical case. J Oral Maxillofac Surg 2011; 69: 2351-2356.

42. Triches TC, Ximenes M, de Souza JG, Neto AR, Cardoso AC, Bolan M. Implant-supported oral rehabilitation in child with Ectodermal dysplasia - 4-year follow-up. Bull Tokyo Dent Coll 2017; 58: 49-56.

43. Becktor KB, Becktor JP, Keller EE. Growth analysis of a patient with ectodermal dysplasia treated with endosseous implants: a case report. Int J Oral Maxillofac Implants 2001; 16: 864-874.

44. Stanford CM, Guckes A, Fete M, Srun S, Richter MK. Perceptions of outcomes of implant therapy in patients with ectodermal dysplasia syndromes. Int J Prosthodont 2008; 21: 195-200.

45. Ioannidou-Marathiotou I, Kotsiomiti E, Gioka C. The contribution of orthodontics to the prosthodontic treatment of ectodermal dysplasia: a long-term clinical report. J Am Dental Assoc 2010; 141: 1340-1345.

46. Rachmiel A, Turgeman S, Emodi O, Aizenbud D, Shilo D. Management of severely atrophic maxilla in ectrodactyly ectodermal dysplasia-cleft syndrome. Plast Reconstr Surg Glob Open 2018; 6: e1678.

47. Hsieh YL, Razzoog M, Garcia Hammaker S. Oral care program for successful long-term full mouth habilitation of patients with hypohidrotic ectodermal dysplasia. Case Rep Dent 2018; 2018 4736495.

48. Dogan MS, Akbaba MH, Yavuz İ, et al. Oral rehabilitation of patients with ectodermal dysplasia: cases series. Int J Health Sci 2016; 4: 59-68.

49. Hanisch M, Wiemann S, Bohner L, Kleinheinz J, Jung S. Association between oral health-related quality of life in people with rare diseases and their satisfaction with dental care in the health system of the Federal Republic of Germany. Int J Environ Res Public Health 2018; 15: 1732

50. Deo K, Sharma YK, Shah B, et al. Improvement in the quality of life of a patient of ectodermal dysplasia with reconstructive surgeries. J Cutan Aesthet Surg 2019; 12: 244-247. 